\section{Peter Schaar erhält den GDD-Datenschutzpreis 2013}

Im Rahmen des Abendprogramms der 37. DAFTA ehrte die Gesellschaft für Datenschutz und Datensicherheit e.V. am 15.11.2013 Herrn Peter Schaar, den Bundesbeauftragten für den Datenschutz und die Informationsfreiheit (BfDI), mit dem Datenschutzpreis der Gesellschaft für Datenschutz und Datensicherheit 2013.

Überreicht wurde dem Preisträger Peter Schaar der vom Kölner Bildhauer und Medaillenschneider Heribert Calleen gestaltete Preis in Form einer Medaille von Prof. Dr. Schwartmann, dem Vorstandsvorsitzenden der GDD.

Mit der Preisverleihung würdigt die GDD Herrn Schaars außerordentlichen und öffentlichkeitswirksamen Einsatz für das Grundrecht auf informationelle Selbstbestimmung, den er vor und während seiner zehnjährigen Amtszeit gezeigt hat.

Zu seinen Erfolgen während seiner Amtszeit als BfDI gehört nicht nur die kritische und zu diversen Diskussionen Anstoß gebende Begleitung zahlloser Gesetzgebungsverfahren, wie - um exemplarisch einige herauszugreifen - dem eGovernment-Gesetz, der TKGNovellierung, dem Gesetz zur Internetsperre und vielen anderen mehr, sondern auch die Schaffung neuer, interaktiver Plattformen zur Bürgerbeteiligung und Informationsbeschaffung, wie die Veröffentlichung des Datenschutzforums, des BfDI-Blogs sowie des Datenschutz-WIKIs, die Bürgerinnenen und Bürgern Möglichkeiten eröffneten, die Fortentwicklung und Anwendung des Datenschutzes interaktiv zu erfahren oder über sich über aktuelle Fragen des Datenschutzes auszutauschen.

Selbst Widrigkeiten, wie bspw. die finanzielle Ausstattung der ihm unterstehenden Behörde oder "Gegenwind" aus höchsten Regierungskreisen konnten ihn nicht davon abbringen, beharrlich sein Ziel zu verfolgen - dem Wirken und Streben nach einem risikoorientieten Datenschutz.

Herr Schaar, so Prof. Schwartmann, hat neue und hohe Maßstäbe für die Arbeit eines Bundesbeauftragten für den Datenschutz gesetzt.

\section{BfDI: Bericht zu Konsequenzen der Abhöraktivitäten der NSA an den Bundestag}

Anlässlich der Sitzung des Deutschen Bundestags am 18.11.2013 zu den Abhöraktivitäten des USamerikanischen Nachrichtendienstes NSA und den Auswirkungen auf Deutschland und die transatlantischen Beziehungen hat der Bundesbeauftragte für den Datenschutz und die Informationsfreiheit Peter Schaar den Abgeordneten des Deutschen Bundestags einen Bericht vorgelegt, der den Abgeordneten des Deutschen Bundestags Anhaltspunkte für mögliche Entscheidungen und Weichenstellungen liefern soll.
Die Kernaussagen des Berichts lauten:

- Grundrechtsschutz und Sicherheit müssen insbesondere im Bereich der Nachrichtendienste in einem ausgewogenen Verhältnis stehen. Dies setzt eine effektive und lückenlose unabhängige Kontrolle nachrichtendienstlicher Tätigkeiten voraus.

- Die berichteten anlasslosen Massendatenerhebungen sind schnell, umfassend, detailliert und - soweit rechtlich zulässig auch öffentlich aufzuklären.

- Strukturelle und/oder regelungstechnische Defizite sind unverzüglich und nachhaltig zu beseitigen - auf nationaler wie internationaler Ebene.

- Die Zusammenarbeit deutscher mit ausländischen Nachrichtendiensten darf nicht dazu führen, durch Aufgabenteilung nationale (verfassungs-)rechtliche Beschränkungen für ihre Tätigkeit zu umgehen („Befugnis-Hopping“).

- Aufgrund der Gesetzgebungs- und Kontrollkompetenz des Deutschen Bundestages über die Nachrichtendienste des Bundes ist eine engere Kooperation der parlamentarisch bestellten Kontrollorgane und die Beseitigung bestehender Kontrolldefizite dringend erforderlich.

Der vollständige Bericht,Abhöraktivitäten US-amerikanischer Nachrichtendienste in Deutschland - Bericht an den Deutschen Bundestag gemäß § 26 Absatz 2 Satz 3 BDSG' steht auf der Internetseite des Bundestages zur Verfügung: $h t t p: / / d i p 21 . b u n d e s t a g . d e /$ dip21/btd/18/000/1800059.pdf

\section{Verbraucherdialog „Mobile Payment"}

Mobiles Bezahlen bedeutet, dass Bezahlvorgänge durch mobile elektronische Techniken initiiert, autorisiert oder realisiert werden. Es gibt unterschiedliche Ansätze und Lösungen. Die Zukunft des Mobile Payment wird vor allem im Einsatz des Smartphones als „mobile Geldbörse" gesehen: Während eine Applikation auf dem Smartphone oder an der Bezahlstelle das bargeldlose Bezahlen ermöglicht, werden die dazu notwendigen Daten mittels drahtloser Übertragungstechnologie, zum Beispiel per NFC-Nahfunktechnik, übertragen. Aber auch der QR-Code wird teilweise für Bezahlvorgänge benutzt. Bargeld, Kreditkarten, Rabattkarten, Gutscheine, Tickets u.a. könnten beim Mobile Payment durch ein Gerät ersetzt werden, um Produkte und Dienstleistungen aller Art zu bezahlen. Bislang wird Mobile Payment vor allem bei Einkäufen mit vergleichsweise geringen Warenwerten angeboten.

Doch wie sicher sind mobile Bezahlverfahren? Wer hat Zugriff auf welche Daten? Wo, wie und zu welchen Zwecken werden Daten gespeichert? Wie erfolgen Nutzerregistrierung und -authentifizierung, wie Bestätigung von Bezahlvorgängen und Kostenkontrolle? Wer haftet im Schadensfall? Und: Wie transparent sind mobile Bezahlverfahren für den Verbraucher, welche Informationen sind erforderlich?

Um diese und weitere verbraucherrelevante Fragen zu diskutieren, hat Verbraucherschutzminister Jochen Hartloff mit einer Auftaktveranstaltung im April 2013 in Mainz den 3. Verbraucherdia- 